NOTICE: This is the author's version of a work that was accepted for publication in Computers \& Chemical Engineering. Changes resulting from the publishing process, such as peer review, editing, corrections, structural formatting and other quality control mechanisms may not be reflected in this document. Changes may have been made to this work since it was submitted for publication. A definitive version was subsequently published in Computers \& Chemical Engineering, Volume 60, 10 January 2014, Pages 231-241. http://dx.doi.org/10.1016/j.compchemeng.2013.09.008 


\title{
A Phenomenological Model of the Mechanisms of Lignocellulosic Biomass Pyrolysis Processes
}

\author{
Abhishek Sharma ${ }^{1}$, Vishnu Pareek ${ }^{\mathbf{1}}$, Shaobin Wang ${ }^{1}$, Zhezi Zhang ${ }^{2}$, Hong Yang $^{2}$ and \\ Dongke Zhang ${ }^{2}$ \\ ${ }^{1}$ Department of Chemical Engineering \\ Curtin University \\ Kent Street, Bentley, Perth, WA 6102, Australia \\ ${ }^{2}$ Centre for Energy \\ The University of Western Australia \\ 35 Stirling Highway, Crawley, WA 6009, Australia
}

\begin{abstract}
A comprehensive particle scale model for pyrolysis of biomass has been developed by coupling the reaction mechanisms and transport phenomena. The model, which also accounts for the combined effect of various parameters such as particle shrinkage and drying, was validated using available experimental data from the literature. The validated model was then used to study the effect of operating temperature and biomass particle size, both of which strongly influenced the rate of biomass conversion. For example, for particle sizes less than 1 $\mathrm{mm}$, a uniform temperature throughout the particle was predicted, thus leading to higher conversion rates in comparison to those in the larger particles. On the other hand, any increase in moisture content led to considerable decrease in the rate of biomass conversion. For the operating conditions considered in this study, the volumetric particle shrinkage also increased the decomposition of biomass to end products.
\end{abstract}

Keywords: Biomass; Biochar; Kinetics; Modelling; Pyrolysis; Tar

\begin{tabular}{llll}
\hline \multicolumn{2}{l}{ Nomenclature } & \multicolumn{2}{l}{ Surface emissivity } \\
$A$ & Pre-exponential factor $\left(\mathrm{s}^{-1}\right)$ & \multicolumn{2}{l}{ Subscript } \\
$C_{p}$ & Specific heat $\left(\mathrm{J} / \mathrm{kg}-{ }^{\circ} \mathrm{C}\right)$ & $B$ & Biomass \\
$D$ & Diffusivity $\left(\mathrm{m}^{2} / \mathrm{s}\right)$ & $T 1$ & Primary tar \\
$E$ & Activation energy $(\mathrm{J} / \mathrm{kmol})$ & $T 2$ & Secondary tar \\
$M$ & Molecular weight $(\mathrm{kg} / \mathrm{kmol})$ & $G$ & Non-condensable gases \\
$P$ & Pressure (atm) & $C$ & Char $/$ Biochar \\
$r_{p}$ & Particle radius/ half-thickness $(\mathrm{m})$ & $W$ & Water vapour \\
$T$ & Temperature $\left({ }^{\circ} \mathrm{C}\right)$ & &
\end{tabular}

* Corresponding Author. Tel : (+618) 9266 4687, Fax: (+618) 9266 2681, Email: v.pareek@curtin.edu.au 


\begin{tabular}{llll}
\hline$v$ & Vapour velocity $(\mathrm{m} / \mathrm{s})$ & $M$ & Moisture \\
$V$ & Volume $\left(\mathrm{m}^{3}\right)$ & $I$ & Inert gas \\
$\varepsilon$ & Particle voidage/porosity & $v$ & Vapour phase \\
$\mu$ & Viscosity $(\mathrm{kg} / \mathrm{m}-\mathrm{s})$ & $s$ & Solid phase \\
$\beta$ & Permeability $\left(\mathrm{m}^{2}\right)$ & Unit conversion \\
$\rho$ & Density $\left(\mathrm{kg} / \mathrm{m}^{3}\right)$ & ${ }^{\circ} \mathrm{C}=$ Kelvin -273.15 \\
$k$ & Thermal conductivity $\left(\mathrm{W} / \mathrm{m}-{ }^{\circ} \mathrm{C}\right)$ & atm $=1.013 \times 10^{5}$ Pascal \\
\hline
\end{tabular}

\section{Introduction}

Owing to their possible effect on the global warming, there is a worldwide drive to reduce our reliance on fossil fuels, which contribute about $98 \%$ of carbon emissions (Demirbaş, 2006). Also, there is a shift from non-renewable energy sources to bio-energy (bio-fuels) due to continuous depletion of fossil fuels. Biofuels are derived from biomass, which significantly decreases emissions of harmful gases such as $\mathrm{SO}_{\mathrm{x}}$ and $\mathrm{NO}_{\mathrm{x}}$ (Zhang et al., 2007). Examples of commonly used biomass include plant matter such as forest residues (dead trees, branches and tree stumps), yard clippings, wood chips and municipal solid waste. The benefit of using biomass arises due to its renewable nature and ability to re-utilize the emitted greenhouse gas $\left(\mathrm{CO}_{2}\right)$. Biomass-derived fuels are currently estimated to contribute around $13 \%$ of the world's energy supply (Demirbas et al., 2009). Pyrolysis is one of several processes for producing energy from biomass (Chakravarti et al., 2012), where a set of thermo-chemical decomposition processes are used to convert the organic materials in biomass into carbon-rich solid and volatile matters by heating in the absence of oxygen (Demirbas \& Arin, 2002). The solid content of pyrolysis products is known as the biochar or char, and is generally high in carbon content. The volatile contents are partly condensed to give a liquid fraction called tar or bio-oil (high molecular weight compounds) along with a mixture of the non-condensable gases $\left(\mathrm{H}_{2}, \mathrm{CO}, \mathrm{CO}_{2}\right.$, and $\mathrm{C} 1-\mathrm{C} 4$ hydrocarbons). The formation of these products is from both primary decomposition of the solid biomass as well as secondary reactions of condensable volatile organic products into low-molecular weight gases, secondary tar and char, while transporting through particle and reactor gas environment (Di Blasi, 2008). The proportion in which liquid and solid products are formed is dictated by not only feedstock properties but also the operating conditions.

Due to the increasing applications of biomass pyrolysis for value added products formation, several modelling studies have been reported in the literature. Thurner and Mann (1981) developed a model for investigating the kinetics of gas, char and tar formation from the pyrolysis of wood. Di Blasi and Branca (2001) examined the kinetics of isothermal primary degradation of beech wood in temperature range of $300-435^{\circ} \mathrm{C}$. They (Di Blasi \& Branca, 2001) found that variation in the product yields and kinetic rates was mainly because of the effect of different heating rates, operating temperatures and experimental setups. Liden et al. (1988) proposed a kinetic model for production of organic liquids from flash pyrolysis of biomass. In their model, it was assumed that wood or other biomass decomposes according to two parallel reactions yielding gas with char, and liquid tar which further decomposes by secondary homogeneous reactions into gaseous products. Some more studies had been undertaken for analysing the secondary tar cracking reactions during biomass pyrolysis 
(Baumlin et al., 2005; Boroson et al., 1989b; Fagbemi et al., 2001; Font et al., 1990; Morf et al., 2002).

For studying the dynamics of pyrolysis process, different models (Chan et al., 1985; Di Blasi, 1993b; Kansa et al., 1977; Koufopanos et al., 1991) had been proposed for understanding transport phenomena with chemical kinetics inside biomass particle. Di Blasi (1996) proposed a transport model for studying the effect of particle shrinkage on pyrolysis. Some assumptions such as negligible moisture content and no condensation of tar species inside the particle were also taken into account (Di Blasi, 1996). Bryden and Hagge (2003) analysed the pyrolysis of a moist, shrinking biomass particle in their model. The authors combined the impact of moisture and particle shrinkage on pyrolysis times and product yields were taken into account. Although this model included the effect of shrinkage due to char, it ignored the shrinkage due to the volume occupied by volatiles (Di Blasi, 1996). Park et al. (2010) studied the degradation mechanism of wood at different temperatures. In this model, the major emphasis was on the endo/exothermicity of the reactions occurring during the process, and also to the effect of pressure generation on particle structure. However, this model didn't account for particle shrinkage and diffusive flux for gaseous species. Lu et al. (2010) proposed a model for studying the effect of particle shape and size on the rate of biomass devolatilization. They contradicted the results of Janse et al. (2000) by stating that spherical particles have slower rate of heat and mass transfer as compared to other aspherical particles of the same volume/mass. Recently, Peters (2011) developed a model for analysing the pyrolysis rate of different biomass samples such as spruce, beech, casuarina, pine wood, cellulose and lignin for different heating rates and particle geometries. However, these models (Lu et al., 2010; Peters, 2011) did not include the effect of particle shrinkage and drying during the pyrolysis.

Although there were currently several models for pyrolysis of biomass particles for predicting the rate of biomass degradation and product yields, most of these models cannot predict the combined effect of all the physical and chemical processes such as moisture content and particle shrinkage. Furthermore, any change in thermo-physical properties of biomass during pyrolysis and catalytic effect of biochar on tar cracking reactions has not been explicitly considered. In this study, a comprehensive model for pyrolysis of biomass particle has been developed, which not only considers the combined impact of process parameters but also includes the catalytic effect of biochar on reaction mechanism.

\section{Model development}

In order to develop a comprehensive model for the pyrolysis of biomass particles, kinetic models for both primary decomposition of biomass and secondary tar cracking reactions as well as momentum, mass and energy balances for biomass degradation were included in the model. Below we briefly describe development of this model under following sub-headings:

\subsection{Kinetic Model}

For the kinetics of reactions, a two-stage reaction model has been proposed by considering biomass as a single homogeneous species instead of a mixture of different components like cellulose, hemicellulose and lignin. During this thermo-chemical degradation, biomass actually decomposes into various products by an infinite number of independent parallel reactions. However, for this study, the reaction products have been lumped into three major classes: gases also known as Non-condensable gas (NCG), high molecular weight organic liquid called as tar (primary and secondary), and a solid residue named as char or biochar (also contains metals/minerals and some amount of ash). 


\subsubsection{Reaction Chemistry}

Any increase in temperature favours dehydration and chain cleavage (like decarboxylation, decarbonylation) reactions of solid biomass, which leads to formation of char, light gases and some amount of water. This also causes the reduction in degree of polymerization of biomass, which further leads to formation of volatiles or primary tar by biomass depolymerization reactions.

The primary tar is mainly comprised of a reactive mixture of oxygenated hydrocarbon compounds, which undergoes reactions such as cracking, partial oxidation and repolymerization homogeneously (in vapour phase) as well as heterogeneously (adsorption on active sites of biochar matrix/surfaces) at higher temperatures. The major products of these reactions are gaseous components and aromatic secondary tars (Mono-aromatic and Polyaromatic hydrocarbons) with some amount of water. This step is based on analysis of homogeneous and heterogeneous primary tar cracking reactions, where the secondary cracking of tar inside the particle results into gaseous products and secondary aromatic tar in temperature range of 227 to $827^{\circ} \mathrm{C}$ (Chan et al., 1985). In a further experimental study, decomposition of sewage sludge at higher temperatures and longer gas residence times in fluidized bed reactor led to increase in non-condensable gases and change in structure of oil compounds due to tar cracking reactions (Shen \& Zhang, 2003).

The aromatic secondary tar also reacts heterogeneously and leads to formation of char by repolymerization reactions at higher temperatures. This step is based on analysis for aromatic tar reactions on catalytic char surfaces. It has been reported in the literature (Sun et al., 2007; Wu et al., 2005; Zhang et al., 2008a; Zhang et al., 2008b) that surfaces of various carbonaceous materials can catalyse the decomposition of hydrocarbons. Based on the experiments performed in temperature range of $400-600^{\circ} \mathrm{C}$, it was found that tar formed by primary pyrolysis of wood is reactive in the presence of wood char causing formation of gaseous components and char (Boroson et al., 1989a). The kinetic parameters for the conversion of tar into char by re-polymerization step inside the biomass particle was also calculated (Di Blasi, 1993a). Hence, it has been deduced that the biochar in the biomass pyrolysis process can catalyse the further decomposition of the tarry products in-situ.

\subsubsection{Reaction Mechanism}

The kinetic reactions for biomass pyrolysis are shown in Figure 1. The primary thermal decomposition of biomass (hemicellulose, cellulose and lignin degradation) leads to formation of gases, primary tar and char (reaction 1, 2 and 3 with kinetic constant $K_{1}, K_{2}$ and $K_{3}$, respectively). Based on earlier theoretical and experimental analysis (Chan et al., 1985; Di Blasi, 1993a; Lu et al., 2010) of these decomposition reactions, they have been considered first order in nature with respect to biomass concentration. The homogeneous and heterogeneous cracking of primary tar, inside the particle and in reactor environment, causes formation of gases and aromatic secondary tar (reaction 4 and 5 with kinetic constant $K_{4}$ and $K_{5}$, respectively); and further heterogeneous cracking of highly aromatic secondary tar leads to production of char (reaction 6 with kinetic constant $K_{6}$ ). Similar to primary biomass decomposition, the secondary tar cracking reactions depend on operating parameters such as temperature. However, there are other parameters such as vapour/gas residence time and available catalytically active sites which affect these reactions. The secondary tar cracking reactions were also considered to be of first order in nature with respect to primary and secondary tar concentration. 


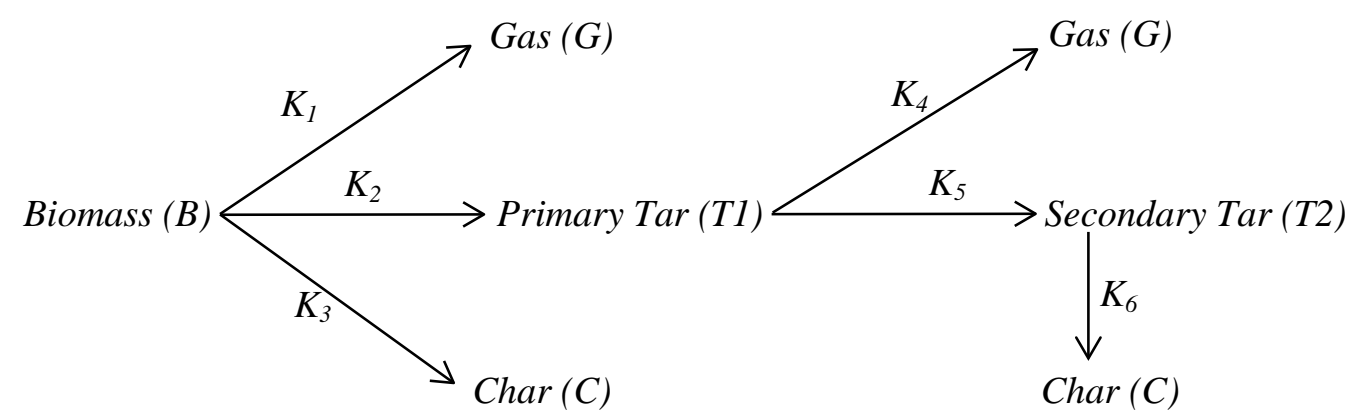

Figure 1 Proposed kinetic model for biomass degradation mechanism.

During thermal decomposition of biomass, the primary step of gas, tar and char formation requires heat energy for bond breaking reactions, and hence, they are endothermic in nature. The secondary tar cracking liberates some amount of energy due to oxidation reactions, and thus, they are slightly exothermic (Koufopanos et al., 1991). The values of kinetic constants in terms of Arrhenius parameters and heat of reactions required during particle modelling have been given in Table 2 and 3, respectively.

\subsection{Particle Model}

A single particle model has been developed for studying transport phenomena with reaction kinetics inside biomass particle. This model considered the heat and mass transfer effects due to formation of temperature and concentration gradients inside particle during decomposition. This model also included the effect of particle drying and shrinkage on biomass pyrolysis, with change in thermo-physical properties of biomass during degradation. For deriving the conservation equations, some assumptions were made for this process:

1. One dimensional, time dependent domain studied for biomass particle.

2. Local thermal equilibrium (no temperature gradient) considered between vapour and solid phase.

3. Re-condensation of volatiles in cooler regions of the particle not taken into account because of higher permeability of char as compared to solid biomass.

4. The volatile or gas phase assumed to follow ideal gas conditions.

5. The kinetic and potential energies of particle were neglected during energy balance.

6. The heat of reactions for NCG and secondary tar formation were considered equal.

7. Particle shape did not change during the degradation process, i.e., cracking or fragmentation not considered.

\subsubsection{Particle drying}

The presence of moisture inside particle affects various physical and chemical processes during decomposition. Moisture content remains mainly as chemically bound water on available absorption sites in biomass or as free water within the pores held by weak capillary forces (Moghtaderi, 2006). Particle drying is an important phenomenon during pyrolysis and governs the rate of mass and heat transfer inside the particle. There are different schemes proposed for considering drying process inside the particle (Bryden et al., 2002). The kinetic scheme proposed by Chan et al. (1985) was utilized in this model for conversion of moisture $(M)$ present in particle to water vapour $(W)$ during pyrolysis process. According to this scheme, water vapour produced during drying is proportional to amount of moisture present inside the particle. This reaction step includes both water of evaporation as well as water of dehydration during the overall process. This is quite a simplified and numerically stable 
scheme, and will lump all the physical processes during drying into a single chemical reaction with kinetic constant $K_{\text {vap }}$ (given in Table 2).

\subsubsection{Particle Shrinkage}

Particle shrinkage is an important physical phenomenon during degradation mechanism. With decrease in particle size during process, temperature gradient as well as residence time of volatiles inside particle gets reduced (Babu \& Chaurasia, 2004), which eventually affects the rate of primary decomposition and secondary tar cracking reactions (Di Blasi, 2002). Different schemes such as Shrinking Core Model and Progressive Conversion Model were proposed for considering the particle shrinkage (Kunii \& Levenspiel, 1991). In the current model, the shrinkage was assumed to be equal to the fraction of the solid which got devolatilized. This was achieved by treating the total particle volume to be a function of the mass of the biomass, moisture and char, and calculating any change in the "solid" volume of the particle both due to conversion of biomass and vaporization of moisture into pyrolysis products (equation 1). However, the particle diameter was treated to be constant throughout the process.

$V=V_{0}+\frac{\left(m_{B}+m_{M}\right)-m_{B, 0}}{m_{C, f}-m_{B, 0}}\left(V_{f}-V_{0}\right)$

here 0 stands for initial state (at start of pyrolysis process) and $f$ stands for final state (at end of process). $m_{B}, m_{M}$ and $m_{C}$ are the mass of biomass, moisture and biochar at any time $t$ during the process, respectively.

Figure 2 shows the mechanism for decomposition of a moist shrinking biomass particle.

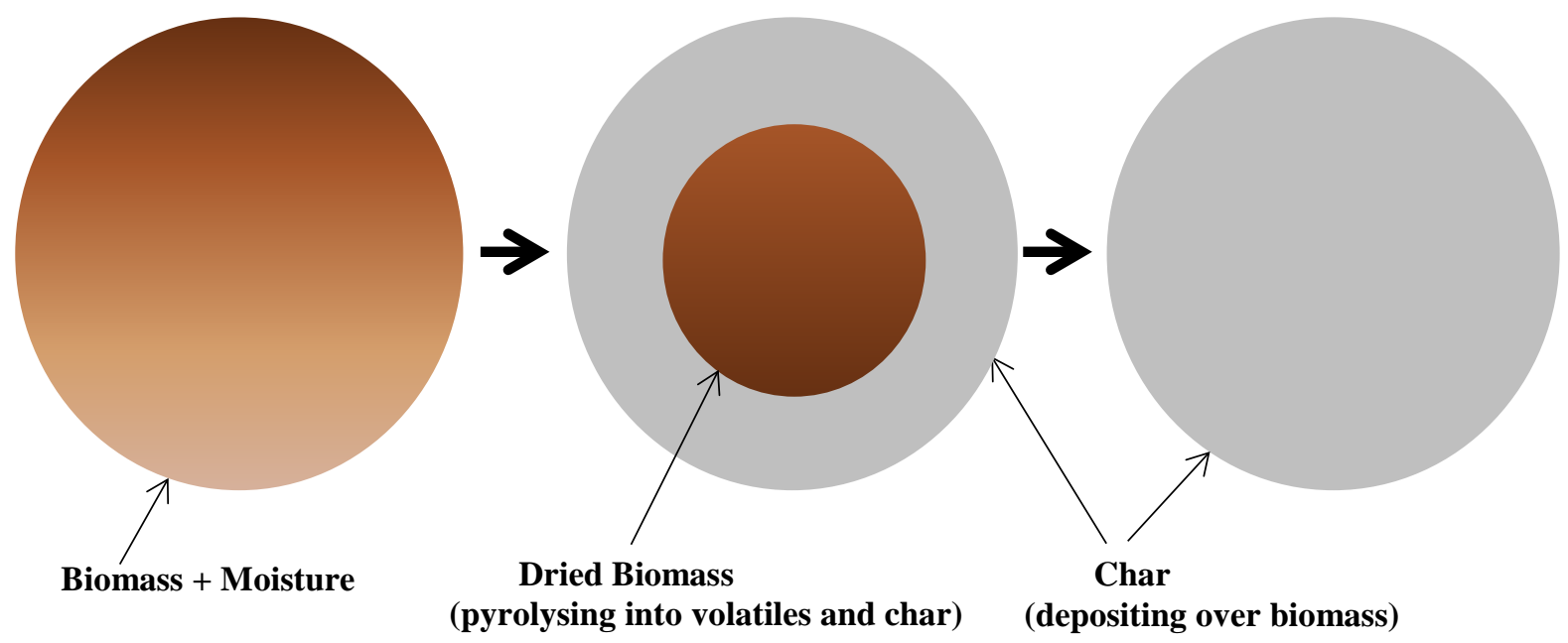

Figure 2 Schematic for showing decomposition progress in a moist shrinking biomass particle.

\subsubsection{Conservation Equations}

Biomass particle acts as a porous medium which allows outflow of vapour products during pyrolysis process. Conservation of momentum (equation 2) in this medium has been given according to modified relationship of Joseph et al. (1982). In this expression, time derivative of velocity has been neglected because it is barely affecting convection studies due to high kinematic viscosity in comparison to permeability to characteristic time of the process inside porous medium. Also, nonlinear drag due to inertial effects has been considered small and hence, replaced with a quadratic drag term. 


$$
\nabla P=-\left(\frac{\mu}{\beta} v+C_{f d} \beta^{-1 / 2} \rho_{v}|v| v\right)
$$

where $\mu$ is the viscosity of vapour phase, $\beta$ is the permeability of porous solid medium [see Appendix A.1.], $C_{f d}$ is form- drag constant (value taken as 0.55), $v$ is the vapour phase velocity in porous medium and $\nabla P$ is the pressure gradient across the medium. In this model, the vapour conditions were calculated using ideal gas equation of state (equation 3).

$P=\rho_{v} R_{g c} T / M_{v}$

here $\rho_{v}$ is the density of the vapour phase, $M_{v}$ is the molecular weight of the vapour phase, $P$ is pressure inside the particle, $T$ is temperature inside the particle and $R_{g c}$ is universal gas constant.

In this model, total eight species have been included for mass balance, that are, biomass, noncondensable gases, primary tar, secondary tar, char, moisture, water vapour and inert gas (such as nitrogen or helium). The mass balance equations are developed for all of these species (equation 4-6 for solid phases and 10-14 for vapour phases) including proposed kinetic model and mechanism for evaporation of moisture during drying of biomass particle.

For solid phase, there are no convective and diffusive transport terms for mass balance. Therefore, the conservation equations for biomass, char and moisture (equation 4-6) are given in terms of source term or rate of production/consumption only.

$$
\begin{aligned}
& \frac{\partial\left(\rho_{B} V\right)}{\partial t}=-\left(K_{1}+K_{2}+K_{3}\right) \rho_{B} V \\
& \frac{\partial\left(\rho_{C} V\right)}{\partial t}=K_{3} \rho_{B} V+K_{6} \varepsilon \rho_{T 2} V \\
& \text { and, } \frac{\partial\left(\rho_{M} V\right)}{\partial t}=-K_{v a p} \rho_{M} V
\end{aligned}
$$

where the left side term of equation is accumulation term for solid phases. Here, $\varepsilon$ is porosity or particle voidage [see Appendix A.1.].

For vapour phase, the conservation equations for all species expressed in this generalized form:

$$
\left[\frac{\partial\left(\varepsilon \rho_{i}\right)}{\partial t}+\nabla \cdot\left(\varepsilon \rho_{i} \vec{v}\right)=-\nabla \vec{J}_{i}+R_{i}\right]
$$

where first and second term on left side of equation refers to accumulation and convective transport due to bulk flow, respectively. Whereas first and second term on right side refers to diffusive transport due to concentration gradient and contribution due to chemical reactions (source term), respectively. Here $\rho_{i}$ is density, $R_{i}$ is rate of production/ consumption by chemical reaction and $\vec{J}_{i}$ is diffusion flux of $i^{\text {th }}$ species in vapour phase given as:

$\vec{J}_{i}=-\varepsilon D_{e f f, i} \nabla \rho_{i}-D_{T, i} \frac{\nabla T}{T}$

where $D_{e f f, i}$ is effective diffusion coefficient [see Appendix A.2.] and $D_{T H, i}$ is thermal (soret) diffusion coefficient of $i^{\text {th }}$ species. As one-dimensional particle domain considered for our model, the above defined conservation equation (equation 7) represented in this model as: 


$$
\left[\frac{\partial\left(\varepsilon \rho_{i}\right)}{\partial t}+\frac{\partial\left(r^{n} \varepsilon \rho_{i} v_{r}\right)}{r^{n} \partial r}=\frac{\partial\left(r^{n} \varepsilon D_{e f f, i} \frac{\partial \rho_{i}}{\partial r}\right)}{r^{n} \partial r}+R_{i}-\frac{\varepsilon \rho_{i}}{V} \frac{\partial V}{\partial t}\right]
$$

The contribution of thermal diffusion is negligible in diffusion flux as compared to molecular diffusion; hence it is neglected in this model (equation 9). The last term on right side is included to consider the effect of volumetric shrinkage of particle on component diffusion. Here $n$ is 0 for flat plate or slab shaped particle, 1 for cylindrical particle, and 2 for spherical particle. $v_{r}$ is the vapour phase velocity in one-dimensional particle domain, calculated using equation 2.

Hence, for Non-condensable Gases $(G)$, the mass conservation equation given as:

$\frac{\partial\left(\varepsilon \rho_{G}\right)}{\partial t}+\frac{\partial\left(r^{n} \varepsilon \rho_{G} v_{r}\right)}{r^{n} \partial r}=\frac{\partial\left(r^{n} \varepsilon D_{e f f, G} \frac{\partial \rho_{G}}{\partial r}\right)}{r^{n} \partial r}+K_{1} \rho_{B}+K_{4} \varepsilon \rho_{T 1}-\frac{\varepsilon \rho_{G}}{V} \frac{\partial V}{\partial t}$

Similarly, for Primary Tar (T1):

$\frac{\partial\left(\varepsilon \rho_{T 1}\right)}{\partial t}+\frac{\partial\left(r^{n} \varepsilon \rho_{T 1} v_{r}\right)}{r^{n} \partial r}=\frac{\partial\left(r^{n} \varepsilon D_{e f f, T 1} \frac{\partial \rho_{T 1}}{\partial r}\right)}{r^{n} \partial r}+K_{2} \rho_{B}-\left(K_{4}+K_{5}\right) \varepsilon \rho_{T 1}-\frac{\varepsilon \rho_{T 1}}{V} \frac{\partial V}{\partial t}$

For Secondary Tar (T2):

$\frac{\partial\left(\varepsilon \rho_{T 2}\right)}{\partial t}+\frac{\partial\left(r^{n} \varepsilon \rho_{T 2} v_{r}\right)}{r^{n} \partial r}=\frac{\partial\left(r^{n} \varepsilon D_{e f f, T 2} \frac{\partial \rho_{T 2}}{\partial r}\right)}{r^{n} \partial r}+K_{5} \varepsilon \rho_{T 1}-K_{6} \varepsilon \rho_{T 2}-\frac{\varepsilon \rho_{T 2}}{V} \frac{\partial V}{\partial t}$

For Water Vapour (W):

$\frac{\partial\left(\varepsilon \rho_{W}\right)}{\partial t}+\frac{\partial\left(r^{n} \varepsilon \rho_{W} v_{r}\right)}{r^{n} \partial r}=\frac{\partial\left(r^{n} \varepsilon D_{e f f, W} \frac{\partial \rho_{W}}{\partial r}\right)}{r^{n} \partial r}+K_{v a p} \rho_{M}-\frac{\varepsilon \rho_{W}}{V} \frac{\partial V}{\partial t}$

and, for Inert Gas (I):

$\frac{\partial\left(\varepsilon \rho_{I}\right)}{\partial t}=\frac{\partial\left(r^{n} \varepsilon D_{e f f, I} \frac{\partial \rho_{I}}{\partial r}\right)}{r^{n} \partial r}-\frac{\varepsilon \rho_{I}}{V} \frac{\partial V}{\partial t}$

For Inert Gas (equation 14), convective flow inside the particle is considered negligible. There is only diffusive flow which governs mass transfer inside the particle.

Based on mass balance equations of vapour phase components (equation 10-14), the overall continuity equation for vapour phase given as:

$\frac{\partial\left(\varepsilon \rho_{v}\right)}{\partial t}+\frac{\partial\left(r^{n} \varepsilon \rho_{v} v_{r}\right)}{r^{n} \partial r}=K_{1} \rho_{B}+K_{2} \rho_{B}-K_{6} \varepsilon \rho_{T 2}+K_{v a p} \rho_{M}-\frac{\varepsilon \rho_{v}}{V} \frac{\partial V}{\partial t}$

For porous biomass particle, the energy balance is given by combining both vapour phase and solid phase in a single conservation equation. The generalized form of this equation is:

$\left[\frac{\partial}{\partial \mathrm{t}}\left(\varepsilon \rho_{v} E_{v}+\rho_{s} E_{S}\right)+\nabla \cdot\left\{\varepsilon \vec{v}\left(\rho_{v} E_{v}+P\right)\right\}=\nabla \cdot\left(k_{e f f} \nabla T-\sum_{i} h_{i} J_{i}-\tau_{e f f} \cdot \vec{v}\right)+S_{R}\right]$

The first and second term on left side of equation 16 corresponds to enthalpy accumulation and energy flow due to convective transport, respectively. Whereas, the first term on right side represents energy contribution due to conduction. Here, $k_{e f f}$ is effective thermal conductivity of medium, $E_{v}$ is total fluid medium energy, $E_{S}$ is total solid medium energy due to thermal inertia of solid medium, and $S_{R}$ is heat of chemical reactions or any other volumetric heat source [see Appendix A.3., A.4. and A.5.]. $\sum_{i} h_{i} J_{i}$ is energy transfer due to 
species diffusion and $\tau_{e f f} . \vec{v}$ is energy transfer due to viscous dissipation. In our model, the energy transfer due to species diffusion and viscous dissipation were considered negligible.

The overall energy conservation equation in terms of specific enthalpies and species concentration given as:

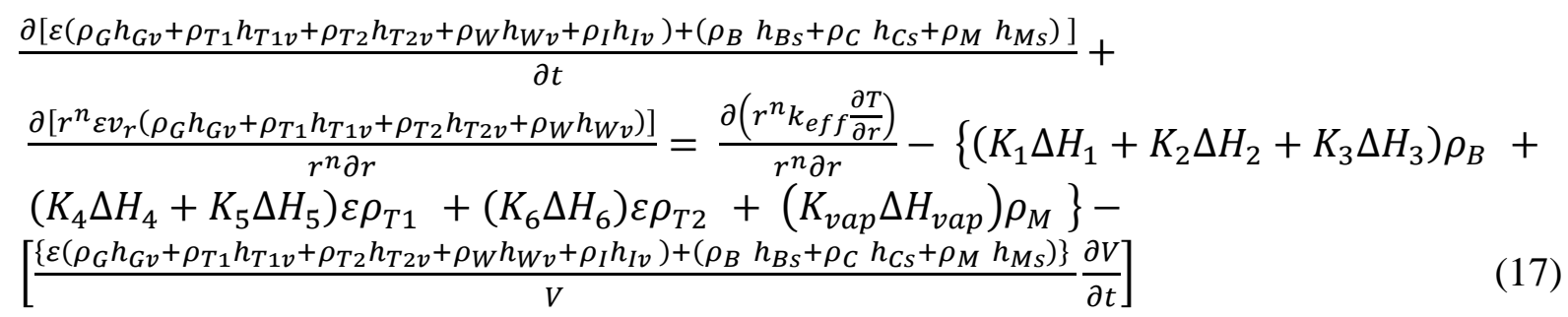

Here, $h_{i v}$ and $h_{i s}$ are specific enthalpies of $i^{\text {th }}$ species in vapour and solid phase, respectively [see Appendix A.4.]. In this equation, the last term on right side represents the effect of particle shrinkage on total energy (both vapour and solid phase enthalpies) during thermal degradation of biomass. The energy accumulation due to pressure forces inside the particle were considered negligible, and therefore, not taken into account.

\section{Numerical Solution}

The momentum, mass and heat balance equations were solved using PDE (partial differential equation) solver pdepe of MATLAB 7.0. This solves initial-boundary value problems for systems of parabolic and elliptic PDEs in the one space variable $r$ and time $t$. The solver converts the PDEs to ODEs (ordinary differential equation) using a second-order accurate spatial discretization based on a specified grid size. The time integration of ODEs was completed using differential-algebraic equation solver of MATLAB for a given time step. The authors are interested to share the details of this MATLAB code with other researchers.

\subsection{Initial and Boundary Conditions}

At time $t=0$, the biomass particle was non - reacting. Therefore, the initial conditions were:

$P(t=0, r)=P_{a t m}=1 \mathrm{~atm}$

$T(t=0, r)=T_{0}=25^{\circ} \mathrm{C}$

$v(t=0, r)=0$

$\rho_{B}(t=0, r)=\rho_{B, 0_{b}}$ (density of moisture-free solid biomass)

$\rho_{M}(t=0, r)=\rho_{M, 0} \quad$ (density of available moisture in biomass)

$\rho_{B, 0}=\rho_{B, 0_{b}}+\rho_{M, 0} \quad$ (total biomass density at initial time)

$\rho_{I}(t=0, r)=0$ (assuming negligible inert gas inside particle at start of process)

$\rho_{C}(t=0, r)=\rho_{G}(t=0, r)=\rho_{T 1}(t=0, r)=\rho_{T 2}(t=0, r)=\rho_{W}(t=0, r)=0$

When biomass was decomposing, the particle boundary conditions were given by conditions of pressure, heat and mass fluxes at certain positions of the particle. In this case, only halfparticle was considered due to symmetrical heating, leading to variation in dependent variables from centre to surface of particle. 
At particle centre:

$\frac{\partial P}{\partial r}(t, r=0)=v(t, r=0)=0$

$\frac{\partial T}{\partial r}(t, r=0)=0$

$\frac{\partial \rho_{G}}{\partial r}(t, r=0)=\frac{\partial \rho_{T 1}}{\partial r}(t, r=0)=\frac{\partial \rho_{T 2}}{\partial r}(t, r=0)=\frac{\partial \rho_{W}}{\partial r}(t, r=0)=\frac{\partial \rho_{I}}{\partial r}(t, r=0)=0$

At particle surface: (considering reactor conditions)

$P\left(t, r=r_{p}\right)=P_{a t m}=1 \mathrm{~atm}$

$-k_{e f f} \frac{\partial T}{\partial r}\left(t, r=r_{p}\right)=h_{\text {heat }}\left(T_{s u}-T_{b u}\right)+\sigma \omega\left(T_{s u}^{4}-T_{w a}^{4}\right)+q_{f u}$

The first component on right side in equation 30 is due to convective heating of particle by inert gas like nitrogen, and the second one corresponds to radiative component arising due to high temperature conditions inside the reactor. The last term on right side is heat flux term for considering the contribution of heat source such as furnace or arc lamp, if provided in the pyrolysis setup. Here, $\omega$ is surface emissivity and $h_{\text {heat }}$ is heat transfer coefficient [see Appendix A.6. and A.7., respectively]. $T_{s u}$ is surface temperature, $T_{b u}$ is bulk gas temperature, $T_{w a}$ is apparatus (reactor) wall temperature and $\sigma$ is Stefan -Boltzmann constant.

$-D_{e f f, i} \frac{\partial \rho_{i}}{\partial r}\left(t, r=r_{p}\right)=h_{m a s s, i}\left(\rho_{i, s u}-\rho_{i, b u}\right)$

where $h_{\text {mass }, i}$ is mass transfer coefficient of $i^{\text {th }}$ species [see Appendix A.7.], $\rho_{i, b u}$ is bulk phase density of $i^{\text {th }}$ species and $\rho_{i, s u}$ is surface density of $i^{\text {th }}$ species. The effect of pressure on diffusive flow is not considered in equation 31. However, the pressure effect in equation 2 is due to the outflowing vapours from inside the biomass particle towards the surface. This pressure difference is used for calculating the vapour velocity which governs the convective flow of volatile components in equations 10 to 13.

\subsection{Thermo-physical properties and Reaction parameters}

For solving the conservation equations, the required values of thermal, physical and chemical properties were taken from literature (Table 1). Also, the kinetic rate constants (Table 2) and heat of reactions (Table 3) for the proposed reaction model were taken from available data.

Table 1 Thermo-physical properties of biomass.

Property Value

References

Biomass Permeability, $\quad \beta_{B, 0 \text { (across) }}: 10^{-14}, \quad \beta_{B, 0 \text { (along) }}: 10^{-11} \quad$ Di Blasi (1998) $\beta_{B, 0}\left(\mathrm{~m}^{2}\right)$

Char Permeability, $\beta_{C, f} \quad \beta_{C, f \text { (across) }}: 5 \times 10^{-12}, \beta_{C, f(\text { along) }}: 5 \times 10^{-11} \quad$ Di Blasi (1998) $\left(\mathrm{m}^{2}\right)$ 
Conductivity, $k_{B, 0}$

$\left(\mathrm{W} / \mathrm{m}-{ }^{\circ} \mathrm{C}\right)$

Char Thermal

Conductivity, $k_{C, f}$

$\left(\mathrm{W} / \mathrm{m}-{ }^{\circ} \mathrm{C}\right)$

Vapour Phase Thermal

$25.77 \times 10^{-3}$

Di Blasi (1998)

Conductivity, $k_{v}$

$\left(\mathrm{W} / \mathrm{m}-{ }^{\circ} \mathrm{C}\right)$

Initial Porosity, $\varepsilon_{B, 0}$

Final Porosity, $\varepsilon_{C, f}$

Molecular Diffusivity,

$D_{i}\left(\mathrm{~m}^{2} / \mathrm{s}\right)$

(considering equal for

all vapour phase

species)

Vapour Phase

Molecular Weight

(kg/kmol)

Heat Capacity ${ }^{\#}$

$\left(\mathrm{J} / \mathrm{kg}-{ }^{\circ} \mathrm{C}\right)$

Initial Emissivity, $\omega_{B, 0}$
Wood : 2300, Char : 1100 , Tar : 1100, NCG: 1100 , Janse et al. (2000) Inert : 1040; Water Vapour : 1996, Moisture : 4180 
Final Emissivity, $\omega_{C, f}$

Initial Pore Diameter,

$d_{\text {poreB }, 0}(\mathrm{~m})$

Final Pore Diameter,

$d_{\text {porec,f }}(\mathrm{m})$

Vapour Phase

Viscosity, (kg/m-s)
1

Branca and Di Blasi

(2003)

$5 \times 10^{-5}$

Grønli and Melaaen

(2000)

$10^{-4}$

Grønli and Melaaen

(2000)

Tar : $3 \times 10^{-5}$, NCG : $3 \times 10^{-5}$, Inert : $3 \times 10^{-5}$

Janse et al. (2000)

Water Vapour : $1.3 \times 10^{-5}$

\# Constant values of heat capacities were taken from literature as a valid assumption for considering thermal effects during biomass particle pyrolysis.

Table 2 Kinetic rate parameters.

\begin{tabular}{llll}
\hline Reaction Constant, $K_{i}$ & Pre-exponential & Activation Energy, $E$ & References
\end{tabular}

Factor, $A$

$(\mathrm{J} / \mathbf{k m o l})$

$\left(\mathrm{s}^{-1}\right)$

$\begin{array}{lll}K_{1} & 4.38 \times 10^{9} & 1.527 \times 10^{8}\end{array}$

(2001)

$\begin{array}{llll}K_{2} & 1.08 \times 10^{10} & 1.48 \times 10^{8} & \text { Di Blasi and Branca }\end{array}$

(2001)

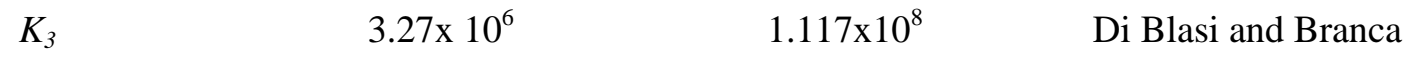

(2001)

$K_{4}{ }^{*} \quad 1.48 \times 10^{6} \quad 1.44 \times 10^{8} \quad$ Chan et al. (1985)

$K_{5}^{*} \quad 1.48 \times 10^{6} \quad 1.44 \times 10^{8} \quad$ Chan et al. (1985) 


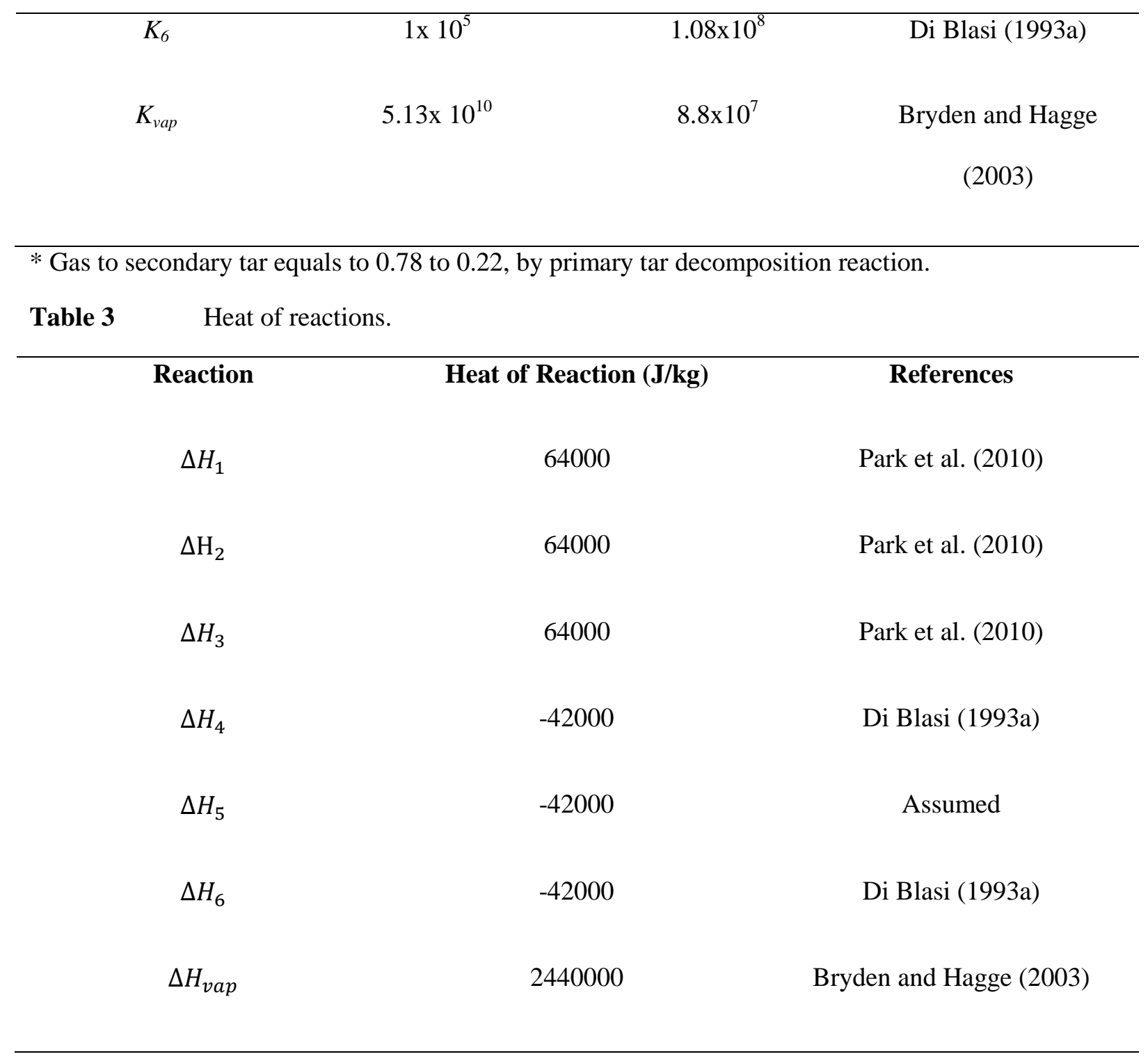

\section{Results and Discussion}

The model results were validated using experimental data available in the literature. The effect of temperature, particle size, moisture content and shrinkage on biomass conversion in different operating conditions has been analysed.

Validation of model results has been done using experimental studies of Sreekanth and Kolar (2009). The conversion time of a $10 \mathrm{~mm}$ diameter and $10 \mathrm{~mm}$ long wood particle (Casuarina equisetifolia) with about $10 \%$ moisture content was analysed in a lab-scale fluidized bed combustor. The density of dry wood particles was $500 \mathrm{~kg} / \mathrm{m}^{3}$. The bed consists of sand particles $\left(550 \mu \mathrm{m}\right.$ size) at a temperature of around $834^{\circ} \mathrm{C}$. According to their analysis (Sreekanth \& Kolar, 2009), the conversion time is inferred as the time at which the dry wood density reduces to $1 \%$ of initial value. This density is considered as the density at particle centre for model analysis. The heat transfer coefficient calculated using correlations given in Appendix A.7 underestimates the rate of heat transfer in fluidized bed conditions. Therefore, for model comparison with existing studies from fluidized systems, heat transfer coefficient values as applicable under respective conditions have been used. In this case, the heat transfer coefficient of $285 \mathrm{~W} / \mathrm{m}^{2}-^{\circ} \mathrm{C}$ (Sreekanth \& Kolar, 2009) has been used. However, the mass transfer rate is not significantly affecting the process, and hence, mass transfer coefficient 
values were kept same as calculated using given correlations in Appendix A.7 for fluidized bed studies.

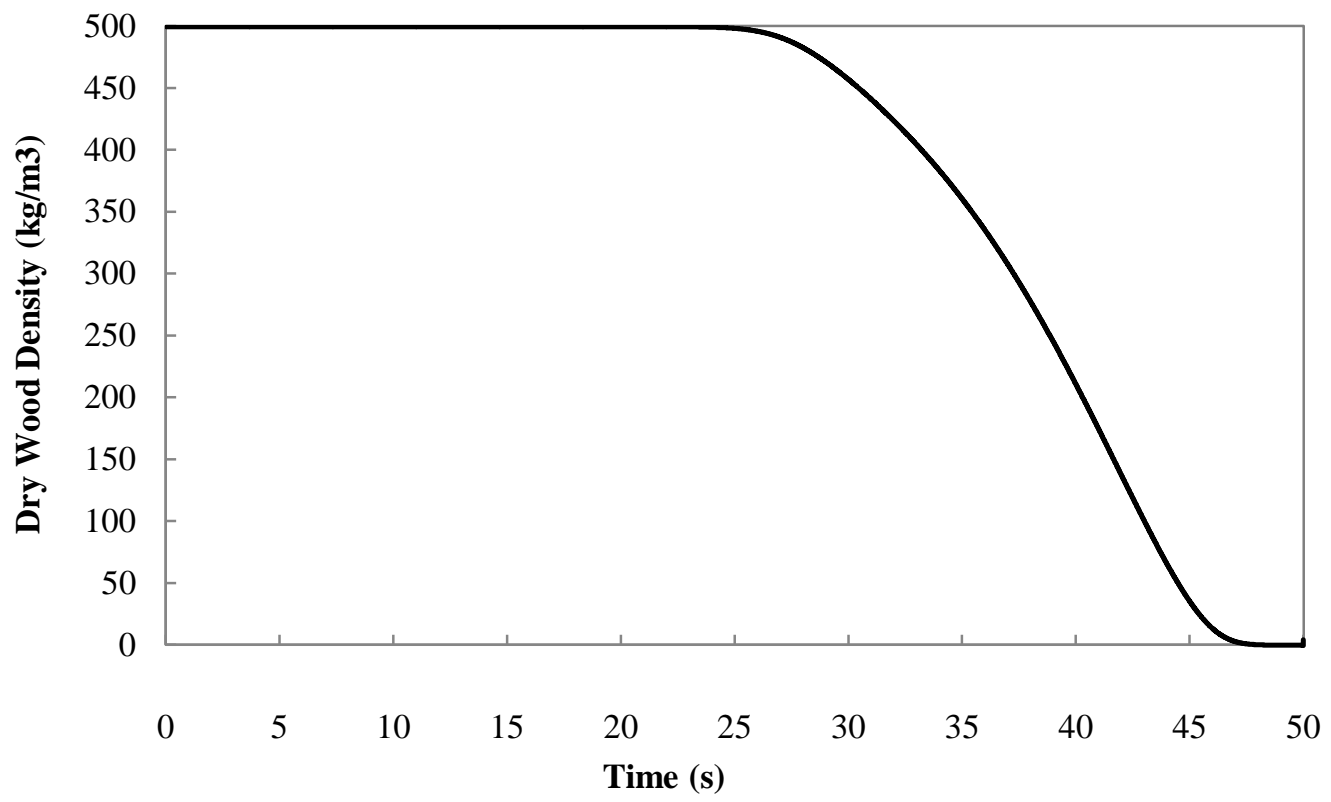

Figure 3 Estimation of conversion time for a moist wood particle in a fluidized bed reactor (particle diameter $=10 \mathrm{~mm}$, reactor temperature $=834^{\circ} \mathrm{C}$ ).

It has been clear from Figure 3 that density at centre starts decreasing after about 26 seconds. The pyrolysis reactions at centre has been started after that time, and led to conversion of wood to char and other volatile products. The density at centre reduces to $1 \%$ of its initial value at around 46.7 seconds. According to experiments (Sreekanth \& Kolar, 2009), this time was coming 46 seconds, which is quite close to the modelling results.

\subsection{Effect of reactor temperature}

The simulation results were compared with experiments of Park et al. (2010) for a spherical biomass particle (moisture free) of $25.4 \mathrm{~mm}$ diameter in temperature range of 415 to $606^{\circ} \mathrm{C}$ in a vertical tube furnace. The results are shown in Figure 4 for three different values of temperature. It was found that results were quite satisfactory in that range, but there were some deviations in mass loss profile with decreasing temperatures. Also, there was negligible decrease in mass of solid at start of process, but thereafter, there was constant rate of mass loss, finally reaching to a fixed (around $20 \%$ here) value of remaining solid product yield. The deviation at lower temperatures was because of incapability of model to predict the devolatilization kinetics, which was mainly due to uncertainties in values of parameters such as activation energies and heat of reactions available in literature for a variety of biomass samples. For large size particles, conductive heat transfer determines the global devolatilization rate at higher temperatures, while the rate is controlled by reaction kinetics at lower temperatures (Chan et al., 1985). Therefore, for analysing the mass loss profile of large length scale particles at lower temperatures, it is highly desired to accurately estimate the reaction kinetic constants of particular biomass samples for getting better results. 


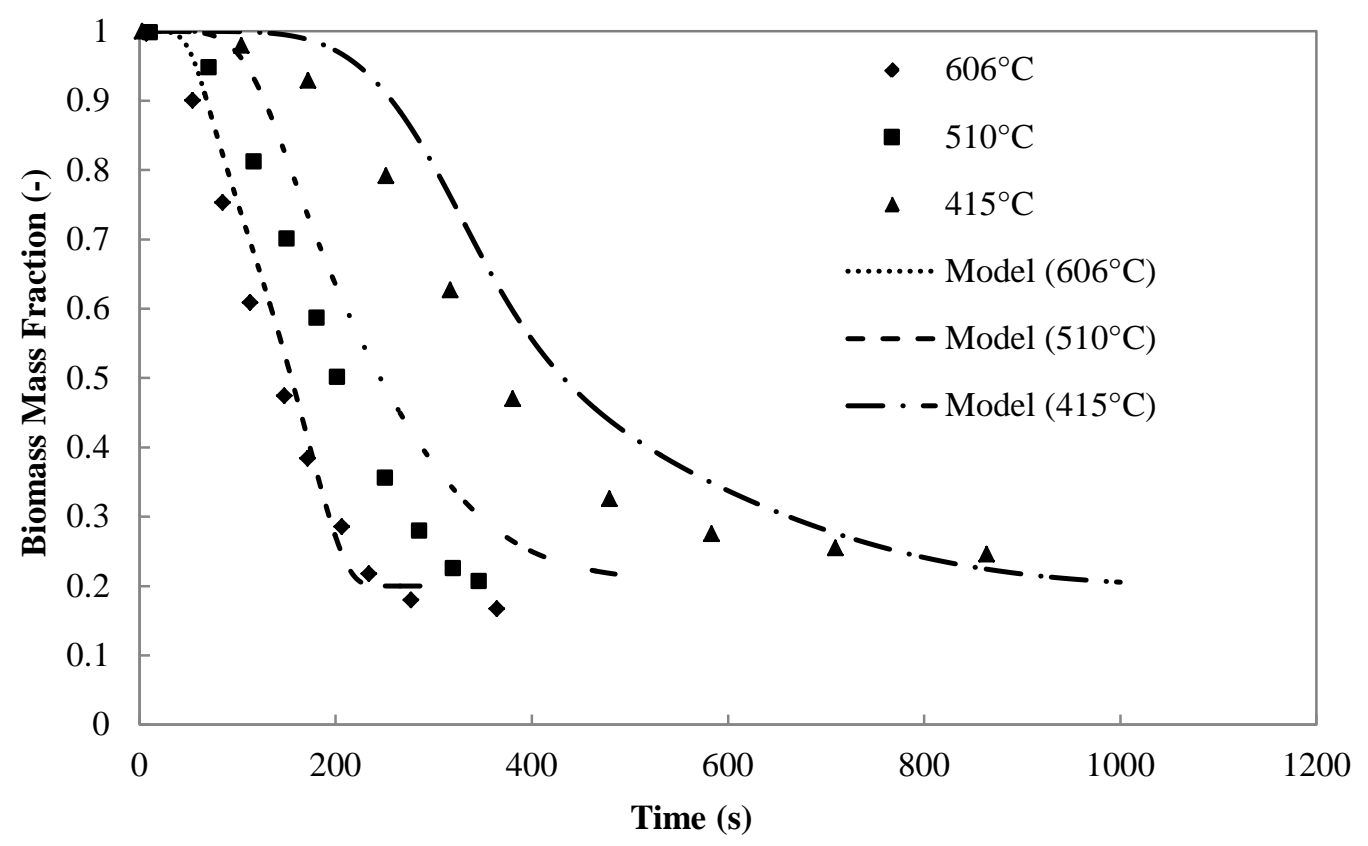

Figure 4 Biomass fraction comparison between proposed model and experiments (Park et al., 2010) for different temperatures (particle diameter $=25.4 \mathrm{~mm}$ ).

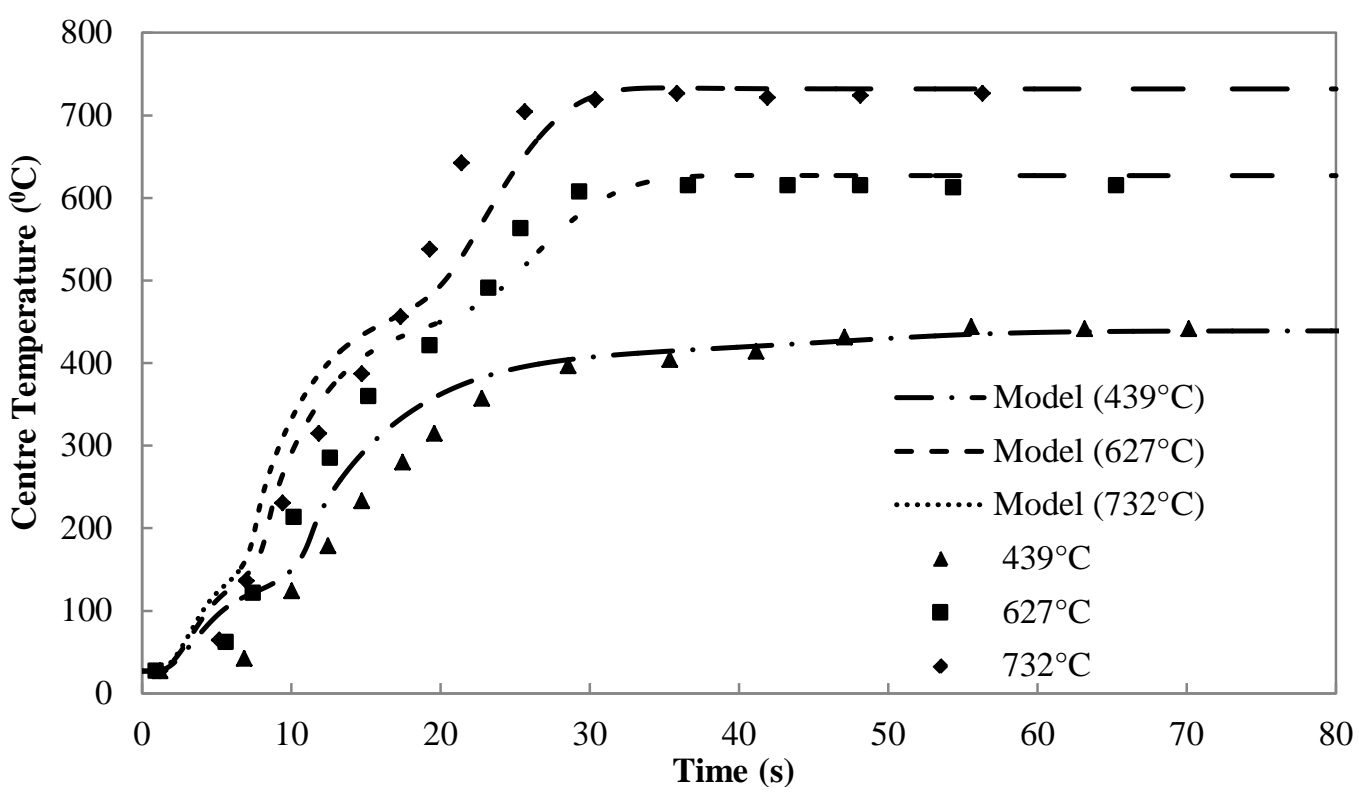

Figure 5 Comparison of particle centre temperature with experiments (Di Blasi \& Branca, 2002) at different temperatures for $4 \mathrm{~mm}$ diameter and $20 \mathrm{~mm}$ length particle.

The simulation results for studying the effect of operating temperature in fluidized bed conditions were compared with experimental analysis of Di Blasi and Branca (2002) using cylindrical beech wood particles of $4 \mathrm{~mm}$ diameter and $20 \mathrm{~mm}$ length (see Figure 5). The thermal conductivity for beech wood particles considered here was $0.209 \mathrm{~W} / \mathrm{m} \mathrm{K}$ across the fibres and $0.349 \mathrm{~W} / \mathrm{m} \mathrm{K}$ along the fibres (Di Blasi and Branca, 2002). For this study, the heat transfer coefficient of $285 \mathrm{~W} / \mathrm{m}^{2}-^{\circ} \mathrm{C}$ (Sreekanth \& Kolar, 2009) has been used. From the figure, it can be seen that modelling results have shown good agreement with experimental values. Different correlations were proposed for studying the conversion time of 
devolatilizing particles (Ross et al., 2000). The characteristic process time (or conversion time) is directly dependent on duration of plateau of particle centre temperature (Di Blasi \& Branca, 2002; Di Blasi et al., 2001). Based on the results, it has been analysed that with increase in outlet temperature, the duration of plateau decreases leading to shorter conversion time of particle. With increase in operating temperature in a reactor configuration for a fixed size particle, the rate of heat transfer inside the particle and rate of chemical reactions increases, leading to reduction in overall time for completion of pyrolysis process. However, temperature has to be chosen with other operating parameters such as particle size and vapour residence time for getting desired product yield, as these parameters affect the rate of secondary tar cracking reactions in reactor environment which lead to variation in product compositions.

\subsection{Effect of particle size}

For analysing the impact of particle size on degradation process, model results were compared with experiments of Di Blasi and Branca (2002) for 3 different diameters of cylindrical beech wood particles (having constant length of $20 \mathrm{~mm}$ ) pyrolyzing at temperature of around $534^{\circ} \mathrm{C}$ in a fluidized sand bed reactor. The thermal conductivity for beech wood particles considered here was $0.209 \mathrm{~W} / \mathrm{m} \mathrm{K}$ across the fibres and $0.349 \mathrm{~W} / \mathrm{m} \mathrm{K}$ along the fibres (Di Blasi and Branca, 2002). In this case, the heat transfer coefficient of $285 \mathrm{~W} / \mathrm{m}^{2}-{ }^{\circ} \mathrm{C}$ (Sreekanth \& Kolar, 2009) has been used. Based on results (shown in Figure 6) for rise in particle centre temperature with time, it was found that with increase in particle diameter, there has been decrease in internal heat transfer rate by conduction. This leads to delay in heating of particle core and increase the overall conversion time of biomass.

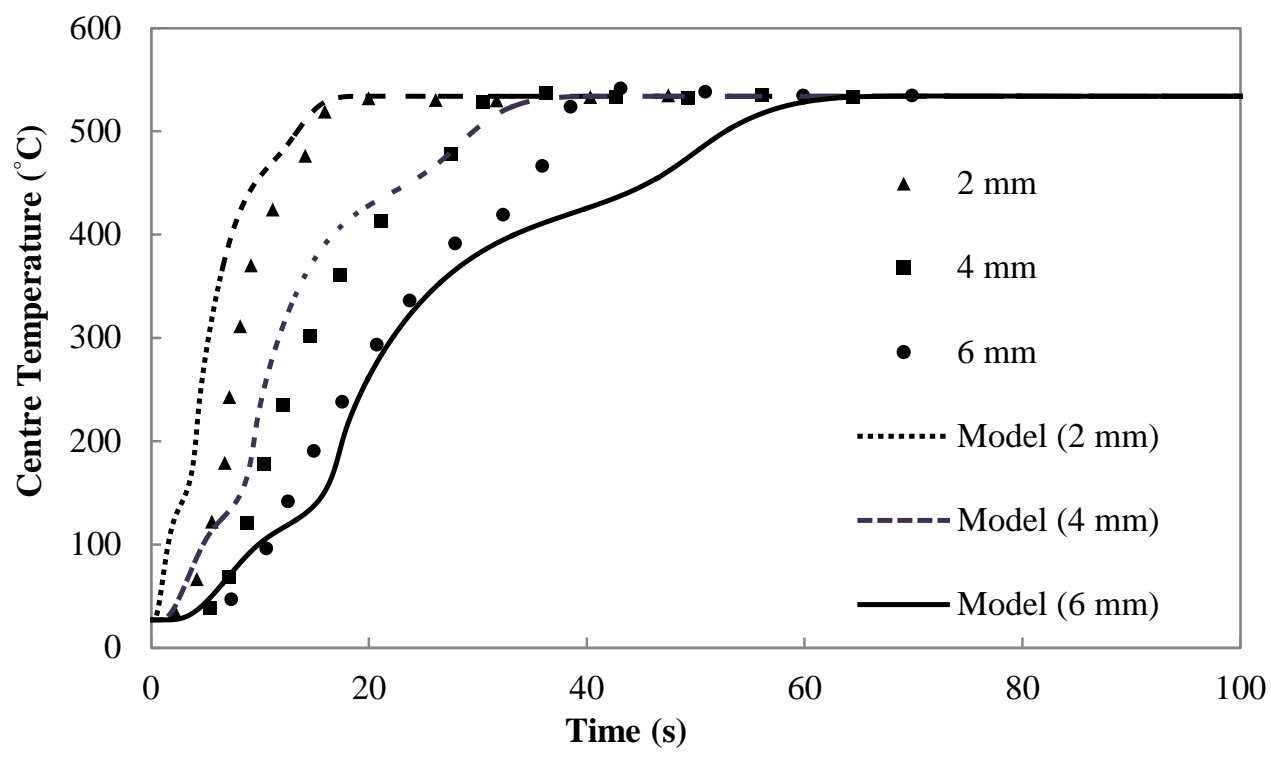

Figure 6 Comparison of particle centre temperature for different sized biomass particles with experiments (Di Blasi \& Branca, 2002) (reactor temperature $=534^{\circ} \mathrm{C}$ ).

Based on modelling comparison between surface and centre temperature for different sized cylindrical particles (as shown in Figure 7) with heat and mass transfer conditions maintained same as of Di Blasi and Branca (2002), it was found that with increase in particle diameter, the heat transfer resistances increase. For $1 \mathrm{~mm}$ particle diameter, temperature gradient between surface and centre is very small, while it increases for $2 \mathrm{~mm}$ and $4 \mathrm{~mm}$ diameter 
particles. Therefore, smaller size biomass particles convert to volatiles and solid products at a faster rate for given operating conditions in any reactor. From the results, it has been also predicted that for particle sizing $<=1 \mathrm{~mm}$, the heat transfer resistances inside the particle can be neglected and a uniform temperature can be assumed throughout the biomass particle during pyrolysis.

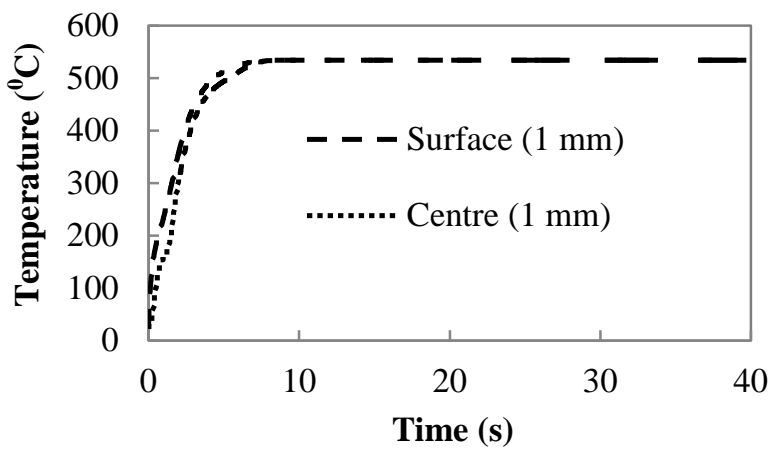

(a)

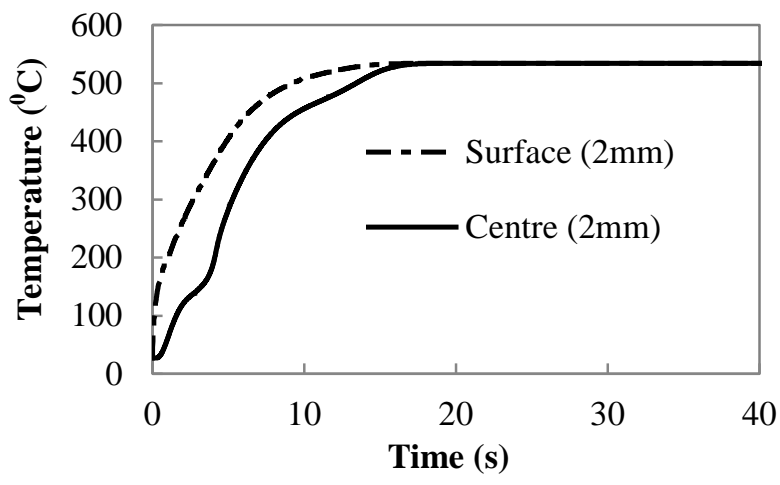

(b)

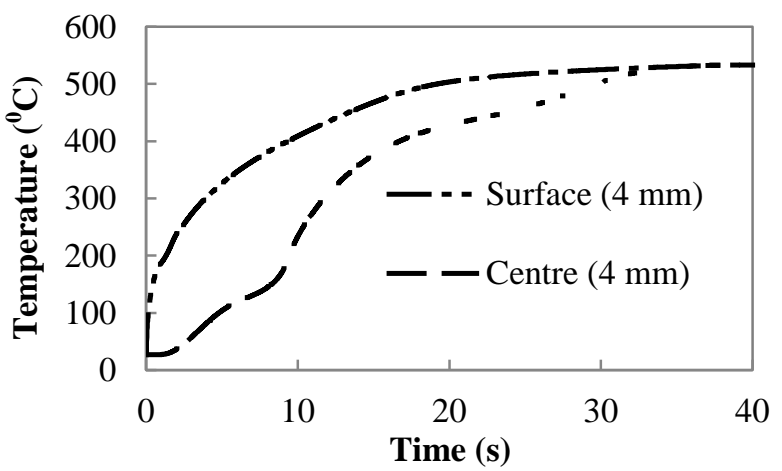

(c)

Figure $7 \quad$ Surface and centre temperature comparison for different sized particles in fluidized bed conditions (reactor temperature $=534^{\circ} \mathrm{C}$ ). (a) particle diameter: $1 \mathrm{~mm}$; (b) particle diameter: 2mm; (c) particle diameter: $4 \mathrm{~mm}$.

Based on the results, it was analysed that with increase in particle size, the biomass conversion rate decreases. However, small size particles favour entrainment and cause incomplete conversion of biomass inside the reactor. This leads to choosing an optimum 
particle size with other operating conditions, such as gas velocity and temperature, for allowing complete conversion of biomass inside the reactor.

\subsection{Effect of moisture content}

Figure 8 shows the effect of moisture content on the biomass conversion during pyrolysis process in a fluidized bed reactor. The operating conditions such as reactor temperature $\left(834^{\circ} \mathrm{C}\right)$ and particle size (diameter $=10 \mathrm{~mm}$, length $=10 \mathrm{~mm}$ ) and heat and mass transfer conditions were kept same as given by Sreekanth and Kolar (2009). It was found that the total time required for completion of the process (dry biomass density at centre $=0$ ) is around 43.6 seconds for $0 \%, 48.1$ seconds for $10 \%$ and 52.8 seconds for $20 \%$ moisture content. With an increase in the moisture content, the time required for evaporation of available water inside the particle increases. This led to the delay in onset of biomass conversion (around 22 seconds for $0 \%$, 26 seconds for $10 \%$ and 30 seconds for 20\%) and hence, increased the overall pyrolysis time for the process.

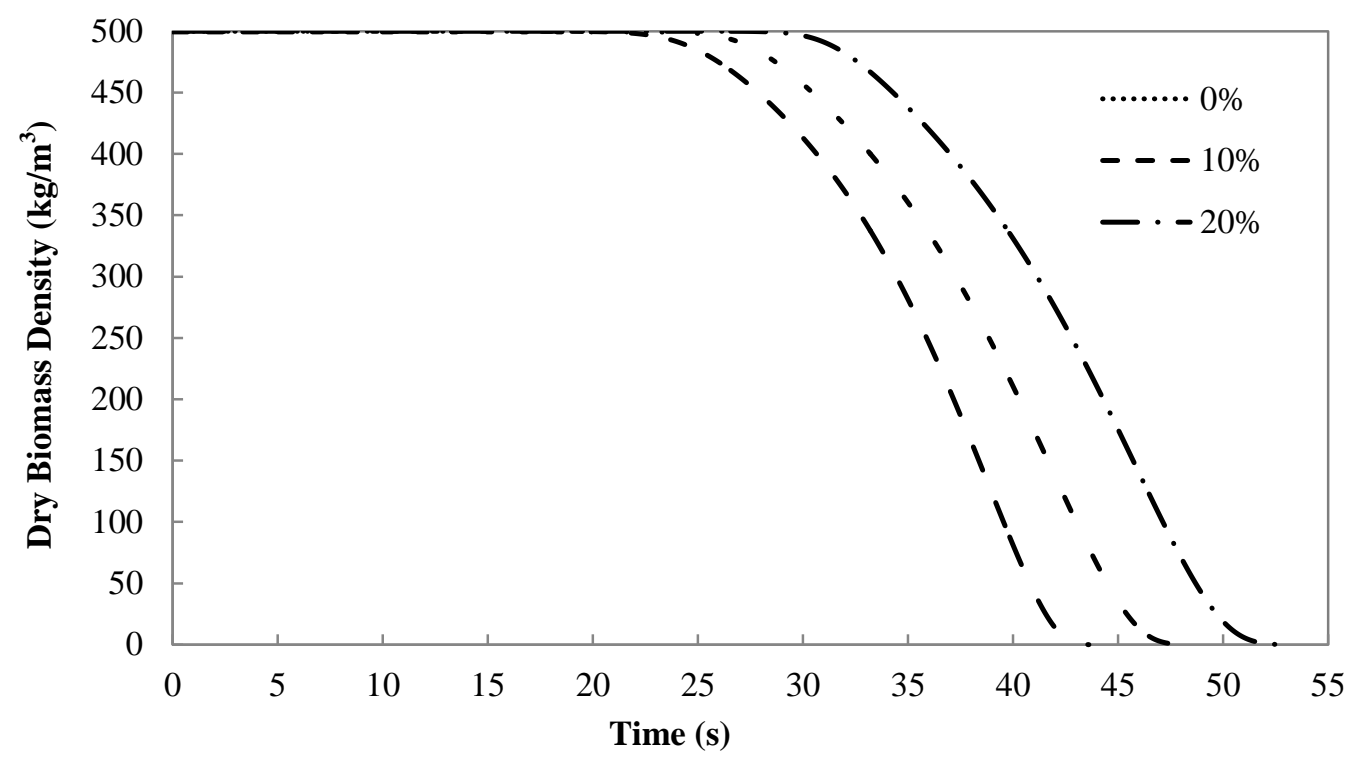

Figure 8 Effect of moisture content on the biomass conversion (reactor temperature = $834^{\circ} \mathrm{C}$, particle diameter $\left.=10 \mathrm{~mm}\right)$.

\subsection{Effect of particle shrinkage}

Figure 9 shows the effect of particle shrinkage on the biomass conversion rate. The operating temperature and diameter of spherical particle was kept at $550^{\circ} \mathrm{C}$ and $25.4 \mathrm{~mm}$, respectively. The heat and mass transfer conditions were considered same as used by Park et al. (2010). It was found that due to particle shrinkage, the rate of conversion of biomass to solid and volatile products increases. This is due to the fact that volumetric shrinkage favours the higher rate of heat transfer which leads to faster decomposition of biomass particle. Therefore, the biomass converted to final products in lesser time for a shrinking particle (about 260 seconds) as compared to a non-shrinking particle (about 390 seconds). 


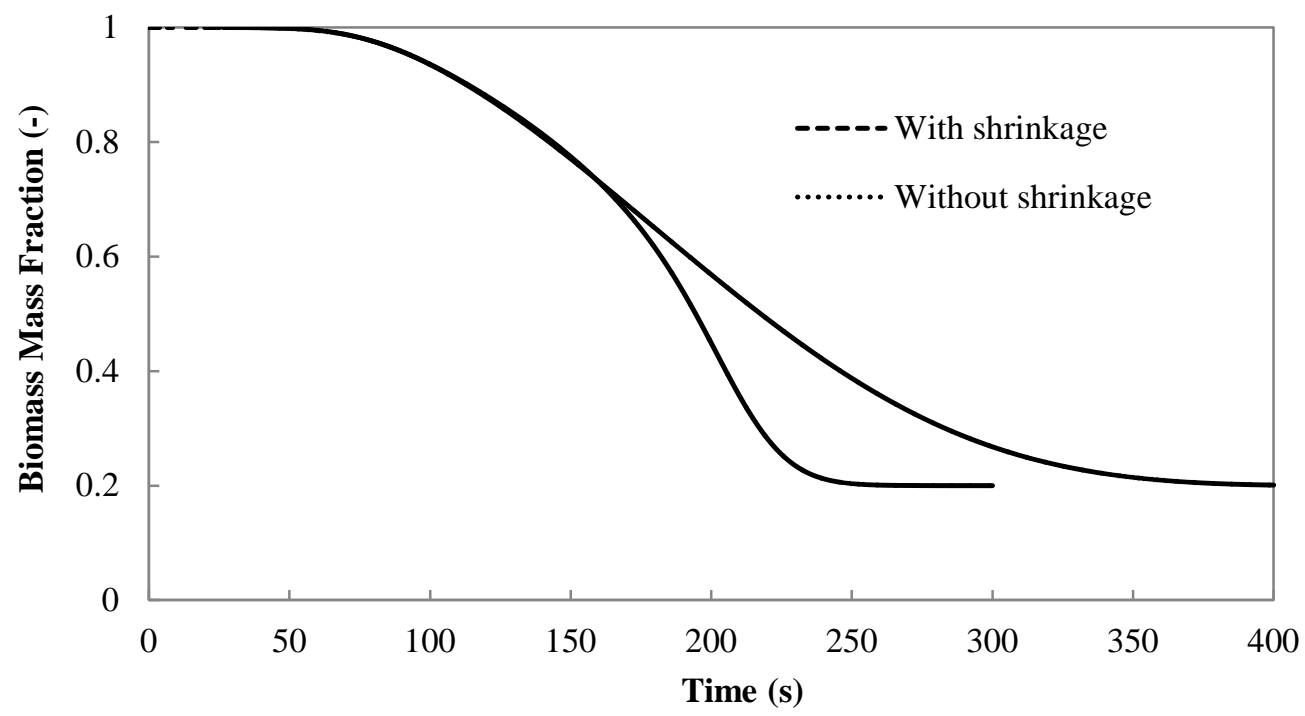

Figure 9 Effect of particle shrinkage on the biomass conversion (operating temperature $=550{ }^{\circ} \mathrm{C}$, particle diameter $=25.4 \mathrm{~mm}$ ).

\section{Conclusions}

A detailed phenomenological model of thermo-chemical decomposition of biomass has been developed. The model includes the kinetics of both the primary solid phase and the secondary gaseous phase (tar) reactions on a lumped basis. The model predictions compared well with the available experimental results for the effect of operating temperature and particle size on the biomass conversion. The effect of the moisture content and particle shrinkage was further analysed and it was found that both parameters significantly affect the rate of biomass decomposition. However, there is a further scope for extending the modelling results for studying the effect of reactor hydrodynamics with operating conditions on product yields in a large-scale reactor.

\section{Acknowledgements}

This research has been partially supported by the Australian Research Council (ARC) under the ARC Linkage projects Scheme (Project \# ARC LP100200135).

\section{Appendix}

\section{A.1. Porosity and Permeability}

There is variation in physical and chemical properties of particle during thermo-chemical conversion of solid biomass into char. Both these properties of biomass also vary with time during conversion and are dependent on apparent densities of biomass, moisture and char:

$$
\begin{aligned}
& \varepsilon=\varepsilon_{B, 0}+\frac{\left(\rho_{B}+\rho_{M}\right)-\rho_{B, 0}}{\rho_{C, f}-\rho_{B, 0}}\left(\varepsilon_{C, f}-\varepsilon_{B, 0}\right) \\
& \beta=\beta_{B, 0}+\frac{\left(\rho_{B}+\rho_{M}\right)-\rho_{B, 0}}{\rho_{C, f}-\rho_{B, 0}}\left(\beta_{C, f}-\beta_{B, 0}\right)
\end{aligned}
$$

Here, 0 stands for initial state (at the start of process) and $f$ stands for final state (at the end of process). 
For considering particle anisotropy, i.e., large variation in some properties of solid biomass and char along and across the grain direction, the permeability for both biomass and char are taken by averaging out their permeability in these directions.

$\beta_{B, 0}=\left(\beta_{B, 0 \text { (across) }}+\beta_{B, 0 \text { (along) })}\right) / 2$

and, $\beta_{C, f}=\left(\beta_{C, f(\text { across })}+\beta_{C, f(\text { along })}\right) / 2$

\section{A.2. Effective Diffusivity}

According to the porous media theory, effective diffusivity, $D_{e f f, i}=\varepsilon * D_{i} / \tau$

where, $D_{i}$ is molecular diffusivity of $i^{\text {th }}$ species ( neglecting the contribution due to Knudsen diffusion) and $\tau$ is tortuosity (taken as 1 in this case)

\section{A.3. Effective Thermal Conductivity}

The effective thermal conductivity of biomass particle is due to contribution of both molecular conductivity and radiative conductivity.

$k_{\text {eff }}=k_{\text {cond }}+k_{\text {rad }}$

where, $k_{\text {cond }}$ is conductive contribution to thermal conductivity $=\varepsilon k_{v}+k_{s}$

$k_{v}$ is vapour phase thermal conductivity.

$k_{s}$ is solid medium thermal conductivity, which is also a function of apparent biomass, moisture and char densities.

$k_{s}=k_{B, 0}+\frac{\left(\rho_{B}+\rho_{M}\right) \rho_{B, 0}}{\rho_{C, f}-\rho_{B, 0}}\left(k_{C, f}-k_{B, 0}\right)$

For considering particle anisotropy, the thermal conductivity for both biomass and char are also taken by averaging out their thermal conductivity across and along the grain direction.

$k_{B, 0}=\left(k_{B, 0 \text { (across) }}+k_{B, 0 \text { (along) }) / 2}\right.$

and, $k_{C, f}=\left(k_{C, f \text { (across) }}+k_{C, f(\text { along })}\right) / 2$

and, $k_{\text {rad }}$ is radiative contribution to thermal conductivity (given as a function of $3^{\text {rd }}$ power of particle temperature)

$k_{\text {rad }}=\frac{4}{1}\left(\operatorname{oed}_{\text {pore }} T^{3}\right)$

where, $d_{\text {pore }}$ is pore diameter (given as a function of apparent densities of biomass, moisture and char)

$$
d_{\text {pore }}=d_{\text {poreB,0}}+\frac{\left(\rho_{B}+\rho_{M}\right) \rho_{B, 0}}{\rho_{C, f}-\rho_{B, 0}}\left(d_{\text {poreC }, f}-d_{\text {pore } B, 0}\right)
$$

and, $e$ is emissivity (value taken as 1).

\section{A.4. Vapour and Solid Phase Enthalpy}

$E_{v}=h_{v}-P / \rho_{v}$

where, $h_{v}=\sum_{i} Y_{i v} h_{i v}$ 
Here, $Y_{i v}$ is mass fraction of $\mathrm{i}^{\text {th }}$ species in vapour phase (also includes water vapour due to particle drying),

$h_{i v}=\int_{T_{o}}^{T} C_{p, i v} d T \quad\left(C_{p, i v}\right.$ is specific heat of $i^{\text {th }}$ species in vapour phase $)$

and, $P$ is pressure inside the particle due to volatile phase

Similarly, $E_{s}=h_{s}=\sum_{i} Y_{i s} h_{i s}$

where, $Y_{i s}$ is mass fraction of $i^{\text {th }}$ species in solid phase

and, $h_{i s}=\int_{T_{o}}^{T} C_{p, i s} d T \quad\left(C_{p, i s}\right.$ is specific heat of $i^{\text {th }}$ species in solid phase $)$

\section{A.5. Heat of Reaction}

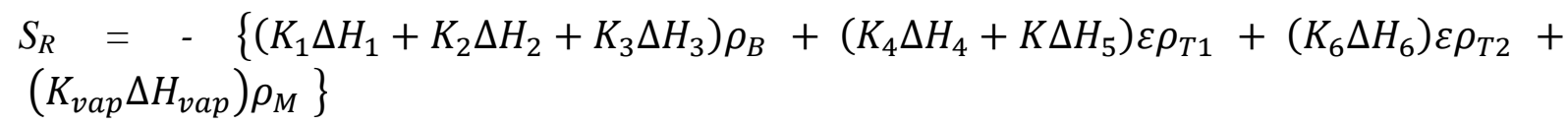

Here, $\Delta H_{1}, \Delta H_{2}$ and $\Delta H_{3}$ are heats of reactions for gas, tar and char formation by primary biomass decomposition, whereas $\Delta H_{4}, \Delta H_{5}$ and $\Delta H_{6}$ are heat of reactions for secondary tar cracking reactions. In this model, the heat energy required for moisture evaporation is also considered, and given as heat of reaction, $\Delta H_{v a p}$ for particle drying during pyrolysis.

\section{A.6. Surface Emissivity}

It is also given as a function of apparent densities of biomass, moisture and char:

$\omega=\omega_{B, 0}+\frac{\left(\rho_{B}+\rho_{M}\right)-\rho_{B, 0}}{\rho_{C, f}-\rho_{B, 0}}\left(\omega_{C, f}-\omega_{B, 0}\right)$

\section{A.7. Heat and Mass Transfer Coefficients}

The heat transfer coefficient can be calculated using correlations available in Bird et al. (2007) for forced convection around submerged objects (flat, cylindrical and spherical shaped) :

For spherical particle,

$N u=2+0.6(\operatorname{Re})^{1 / 2}(\operatorname{Pr})^{1 / 3}$

For cylindrical particle,

$$
N u=\left(0.376(\operatorname{Re})^{1 / 2}+0.057(\operatorname{Re})^{2 / 3}\right)(\operatorname{Pr})^{1 / 3}+0.92[\ln (7.4055 / \operatorname{Re})+4.18 R e]^{-1 / 3}(\operatorname{Re})^{1 / 3}(\operatorname{Pr})^{1 / 3}
$$

For flat or slab shaped particle,

$N u=0.332(\operatorname{Re})^{1 / 2}(\operatorname{Pr})^{1 / 3}$

where, $N u$ (stands for Nusselt Number) $=\frac{h_{h e a t} d_{p}}{k_{g}}$

$R e$ (stands for Reynold Number $)=\frac{d_{p} v_{g} \rho_{g}}{\mu_{g}}$

and, $\operatorname{Pr}$ (stands for Prandtl Number) $=\frac{\mu_{g} c_{p g}}{k_{g}}$ 
here, $d_{p}$ is particle diameter (for cylindrical and spherical shaped) / particle length (for slab shaped)

and, $k_{g}, v_{g}, \rho_{g}, \mu_{g}$ and $c_{p g}$ are thermal conductivity, relative velocity, density, viscosity and heat capacity of surrounding gas, respectively.

The mass transfer coefficient for each species can be calculated using correlations available in Bird et al. (2007), which are similar analogies as that for heat transfer coefficients of given particles.

Like, for spherical particle

$S h=2+0.6(R e)^{1 / 2}(S c)^{1 / 3}$

where, $S h$ (stands for Sherwood Number) $=\frac{h_{m a s s} d_{p}}{D_{\text {eff }}}$

and, Sc (stands for Schmidt Number ) $=\frac{\mu_{g}}{\rho_{g} D_{e f f}}$

\section{References}

Babu, B. V., \& Chaurasia, A. S. (2004). Heat transfer and kinetics in the pyrolysis of shrinking biomass particle. Chemical Engineering Science, 59(10), 1999-2012.

Baumlin, S., Broust, F., Ferrer, M., Meunier, N., Marty, E., \& Lédé, J. (2005). The continuous self stirred tank reactor: measurement of the cracking kinetics of biomass pyrolysis vapours. Chemical Engineering Science, 60(1), 41-55.

Bird, R. B., Stewart, W. E., \& Lightfoot, E. N. (2007). Transport Phenomena (2 ed.). New York: John Wiley and Sons.

Boroson, M. L., Howard, J. B., Longwell, J. P., \& Peters, W. A. (1989a). Heterogeneous cracking of wood pyrolysis tars over fresh wood char surfaces. Energy \& Fuels, 3(6), 735-740.

Boroson, M. L., Howard, J. B., Longwell, J. P., \& Peters, W. A. (1989b). Product yields and kinetics from the vapor phase cracking of wood pyrolysis tars. AIChE Journal, 35(1), 120-128.

Branca, C., \& Di Blasi, C. (2003). Kinetics of the isothermal degradation of wood in the temperature range 528-708 K. Journal of Analytical and Applied Pyrolysis, 67(2), 207-219.

Bryden, K. M., \& Hagge, M. J. (2003). Modeling the combined impact of moisture and char shrinkage on the pyrolysis of a biomass particle. Fuel, 82(13), 1633-1644.

Bryden, K. M., Ragland, K. W., \& Rutland, C. J. (2002). Modeling thermally thick pyrolysis of wood. Biomass and Bioenergy, 22(1), 41-53.

Chakravarti, S., Bonaquist, D. P., Drnevich, R. F., \& Shah, M. M. (2012). Natural gas enhanced biomass to liquids: Project development and modeling. Computers \& Chemical Engineering, 47(0), 6775.

Chan, W.-C. R., Kelbon, M., \& Krieger, B. B. (1985). Modelling and experimental verification of physical and chemical processes during pyrolysis of a large biomass particle. Fuel, 64(11), 1505-1513.

Demirbaş, A. (2006). Global Renewable Energy Resources. Energy Sources, Part A: Recovery, Utilization, and Environmental Effects, 28(8), 779-792.

Demirbas, A., \& Arin, G. (2002). An Overview of Biomass Pyrolysis. Energy Sources, 24(5), 471-482.

Demirbas, M. F., Balat, M., \& Balat, H. (2009). Potential contribution of biomass to the sustainable energy development. Energy Conversion and Management, 50(7), 1746-1760.

Di Blasi, C. (1993a). Analysis of Convection and Secondary Reaction Effects Within Porous Solid Fuels Undergoing Pyrolysis. Combustion Science and Technology, 90(5), 315 - 340. 
Di Blasi, C. (1993b). Modeling and simulation of combustion processes of charring and non-charring solid fuels. Progress in Energy and Combustion Science, 19(1), 71-104.

Di Blasi, C. (1996). Heat, momentum and mass transport through a shrinking biomass particle exposed to thermal radiation. Chemical Engineering Science, 51(7), 1121-1132.

Di Blasi, C. (1998). Physico-chemical processes occurring inside a degrading two-dimensional anisotropic porous medium. International Journal of Heat and Mass Transfer, 41(24), 41394150.

Di Blasi, C. (2002). Modeling intra- and extra-particle processes of wood fast pyrolysis. AlChE Journal, 48(10), 2386-2397.

Di Blasi, C. (2008). Modeling chemical and physical processes of wood and biomass pyrolysis. Progress in Energy and Combustion Science, 34(1), 47-90.

Di Blasi, C., \& Branca, C. (2001). Kinetics of Primary Product Formation from Wood Pyrolysis. Industrial \& Engineering Chemistry Research, 40(23), 5547-5556.

Di Blasi, C., \& Branca, C. (2002). Temperatures of Wood Particles in a Hot Sand Bed Fluidized by Nitrogen. Energy \& Fuels, 17(1), 247-254.

Di Blasi, C., Branca, C., Santoro, A., \& Gonzalez Hernandez, E. (2001). Pyrolytic behavior and products of some wood varieties. Combustion and Flame, 124(1-2), 165-177.

Fagbemi, L., Khezami, L., \& Capart, R. (2001). Pyrolysis products from different biomasses: application to the thermal cracking of tar. Applied Energy, 69(4), 293-306.

Font, R., Marcilla, A., Verdu, E., \& Devesa, J. (1990). Kinetics of the pyrolysis of almond shells and almond shells impregnated with cobalt dichloride in a fluidized bed reactor and in a pyroprobe 100. Industrial \& Engineering Chemistry Research, 29(9), 1846-1855.

Galgano, A., \& Di Blasi, C. (2003). Modeling Wood Degradation by the Unreacted-Core-Shrinking Approximation. Industrial \& Engineering Chemistry Research, 42(10), 2101-2111.

Grønli, M. G., \& Melaaen, M. C. (2000). Mathematical Model for Wood PyrolysisComparison of Experimental Measurements with Model Predictions. Energy \& Fuels, 14(4), 791-800.

Janse, A. M. C., Westerhout, R. W. J., \& Prins, W. (2000). Modelling of flash pyrolysis of a single wood particle. Chemical Engineering and Processing, 39(3), 239-252.

Joseph, D. D., Nield, D. A., \& Papanicolaou, G. (1982). Nonlinear equation governing flow in a saturated porous medium. Water Resour. Res., 18(4), 1049-1052.

Kansa, E. J., Perlee, H. E., \& Chaiken, R. F. (1977). Mathematical model of wood pyrolysis including internal forced convection. Combustion and Flame, 29(311-324.

Koufopanos, C. A., Papayannakos, N., Maschio, G., \& Lucchesi, A. (1991). Modelling of the pyrolysis of biomass particles. Studies on kinetics, thermal and heat transfer effects. The Canadian Journal of Chemical Engineering, 69(4), 907-915.

Kunii, D., \& Levenspiel, O. (1991). Fluidization Engineering (2 ed.). Massachusetts: ButterworthHeinemann.

Liden, A., Berruti, F., \& Scott, D. S. (1988). A kinetic model for the production of liquids from the flash pyrolysis of biomass. Chemical Engineering Communications, 65(1), 207-221.

Lu, H., Ip, E., Scott, J., Foster, P., Vickers, M., \& Baxter, L. L. (2010). Effects of particle shape and size on devolatilization of biomass particle. Fuel, 89(5), 1156-1168.

Moghtaderi, B. (2006). The state-of-the-art in pyrolysis modelling of lignocellulosic solid fuels. Fire and Materials, 30(1), 1-34.

Morf, P., Hasler, P., \& Nussbaumer, T. (2002). Mechanisms and kinetics of homogeneous secondary reactions of tar from continuous pyrolysis of wood chips. Fuel, 81(7), 843-853.

Park, W. C., Atreya, A., \& Baum, H. R. (2010). Experimental and theoretical investigation of heat and mass transfer processes during wood pyrolysis. Combustion and Flame, 157(3), 481-494.

Peters, B. (2011). Validation of a numerical approach to model pyrolysis of biomass and assessment of kinetic data. Fuel, 90(6), 2301-2314.

Ross, D. P., Heidenreich, C. A., \& Zhang, D. K. (2000). Devolatilisation times of coal particles in a fluidised-bed. Fuel, 79(8), 873-883. 
Shen, L., \& Zhang, D.-K. (2003). An experimental study of oil recovery from sewage sludge by lowtemperature pyrolysis in a fluidised-bed. Fuel, 82(4), 465-472.

Sreekanth, M., \& Kolar, A. K. (2009). Progress of conversion in a shrinking wet cylindrical wood particle pyrolyzing in a hot fluidized bed. Journal of Analytical and Applied Pyrolysis, 84(1), 53-67.

Sun, Z. Q., Wu, J., Haghighi, M., \& Zhang, D. K. (2007). Methane Cracking over a Bituminous Coal Char. Energy and Fuels, 21(3), $1601-1605$.

Thurner, F., \& Mann, U. (1981). Kinetic investigation of wood pyrolysis. Industrial \& Engineering Chemistry Process Design and Development, 20(3), 482-488.

Wu, J., Fang, Y., Wang, Y., \& Zhang, D. K. (2005). Combined coal gasification and methane reforming for production of syngas in a fluidised-bed reactor. Energy and Fuels, 19(512-516.

Zhang, Q., Chang, J., Wang, T., \& Xu, Y. (2007). Review of biomass pyrolysis oil properties and upgrading research. Energy Conversion and Management, 48(1), 87-92.

Zhang, Y., Wu, J.-h., \& Zhang, D. K. (2008a). Cracking of Simulated Oil Refinery Off-Gas over a Coal Char, Petroleum Coke, and Quartz. Energy \& Fuels, 22(2), 1142-1147.

Zhang, Y., Wu, J., \& Zhang, D. K. (2008b). Ethane Cracking in Coal Char Bed and Quartz Sand Bed. Petrochemical Technology, 37(8), 770-775. 